NOTE ON A THGONONETRICAL PROOF OF THE ORTHOCENTKE, ETC.

\title{
Note on a trigonometrical proof of the orthocentre property of a triangle.
}

The following argument is simple and instructive, but I do not remember seeing it before.

We start with the identity

$$
\sin ^{2} \alpha+\cos ^{2} \alpha=\sin ^{2} \beta+\cos ^{2} \beta
$$

from which it follows that, for all values of $\alpha, \beta, \gamma$, $(\cos \alpha \cdot \cdots \cos \beta)(\cos \alpha+\cos \beta+\cos \gamma-\cos \gamma)$

$$
+(\sin \alpha-\sin \beta)(\sin \alpha+\sin \beta+\sin \gamma-\sin \gamma)
$$

vanishes.

Hence if the rectangular Cartesian coordinates of four points $A, B, C, P$ are, respectively,

$$
\begin{aligned}
& (\cos \alpha, \sin \alpha), \\
& (\cos \beta, \sin \beta), \\
& (\cos \gamma, \sin \gamma),
\end{aligned}
$$

and

$$
(\cos \alpha+\cos \beta+\cos \gamma, \sin \alpha+\sin \beta+\sin \gamma),
$$

then the line $A B$ is perpendicular to $C P$. Similarly $B C$ is perpendicular to $A P$, and $C A$ to $B P$. Hence $P$ is the orthocentre of the triangle $A B C$.

Conversely every triangle has an orthocentre, since we can apply the above argument to any given triangle by taking the circumcentre 0 of the triangle as origin, and the radius of the circumcircle as unit of measurement.

Again, since the coordinates of $G$ the centroid of $A B C$ are

$$
\left\{\frac{1}{3}(\cos \alpha+\cos \beta+\cos \gamma), \frac{1}{3}(\sin \alpha+\sin \beta+\sin \gamma)\right\},
$$

it follows that $G$ lies in $O P$ and that $3 O G=O P$.

H. W. Turnbulą. 